\title{
Energy manipulation of isonitrogenous diets for broiler chickens
}

\author{
O.F. Akinmoladun ${ }^{1,2 \#} \&$ A.B. Falowo ${ }^{1,3}$ \\ ${ }^{1}$ Department of Livestock and Pasture Science, University of Fort Hare, Alice, Eastern Cape, South Africa \\ ${ }^{2}$ Department of Animal and Environmental Biology, AdekunleAjasin University Akungba-Akoko, Ondo-State, Nigeria \\ ${ }^{3}$ Department of Animal Science, Adekunle Ajasin University Akungba-Akoko, Ondo-State, Nigeria
}

(Submitted 7 August 2019; Accepted 13 March 2020; Published 6 January 2021)
Copyright resides with the authors in terms of the Creative Commons Attribution 4.0 South African Licence.
See: http://creativecommons.org/licenses/by/4.0/za
Condition of use: The user may copy, distribute, transmit and adapt the work, but must recognise the authors and the South African Journal of Animal Science.

\begin{abstract}
A total of 378 unsexed Anak broiler chicks were used to assess the effects of varying energy levels and manipulation on growth, haematology, and carcass traits. The experiment was conducted in two phases. First, one-day-old chicks were randomly assigned to one of three starter diets, which differed in their energy density (LSE: $2786.80 \mathrm{Kcal} / \mathrm{kg}$; OSE: $3015.40 \mathrm{Kcal} / \mathrm{kg}$; and HSE: $3252.20 \mathrm{Kcal} / \mathrm{kg}$ ). The chicks were assigned to seven replicates per treatment with 18 chicks per replicate. When the chicks were 27 days old, they were randomly re-allocated to three finisher diets (LFE: $2770.66 \mathrm{Kcal} / \mathrm{kg}$, OFE: $2961.74 \mathrm{Kcal} / \mathrm{kg}$, and HFE: $3150.43 \mathrm{Kcal} / \mathrm{kg}$ ). Thus, there were seven replicates of nine treatments with six chicks per replicate in the finishing phase. The starter and finisher diets were isonitrogenous. Birds fed the OSE and HSE starter diets gained more weight and were heavier at 27 days than birds fed LSE. Energy intake by birds fed HSE was greater than by birds fed OSE, and birds fed OSE had greater energy intake than birds fed LSE. Feed conversion ratio was improved for birds fed OSE and HSE. Birds fed LSE and then HFE consumed the least feed and gained as much or more weight during the finisher phase as any other group. Overall, FCR between days 27 and 50 tended to differ among the treatments $(P=0.06)$. Total protein intake increased with decreasing dietary energy in both phases. Varying dietary energy levels did not affect the haematological parameters, carcass traits and internal organ weights.
\end{abstract}

Keywords: carcass, compensatory growth, growth, haematology, lipogenesis, skeletal abnormalities Corresponding author: festus.akinmoladun@aaua.edu.ng

\section{Introduction}

In the last few decades, selection of and improvements in nutrition and management of broiler chickens have contributed to their increased growth performance. An increase in average growth rate of about 40 grams has been recorded annually, and the time required to attain market weight $(1.8 \mathrm{~kg})$ has decreased by 0.75 days per year in the past 30 years (Tumova et al., 2002). Such improvement in THE growth rate usually coincides with increased breast muscles, carcass water and fat content (Havenstein et al., 2003). However, the intensity of selection for high performance has resulted in health problems in broiler production (Nansuay et al., 2015). Skeletal abnormalities, cardiovascular diseases, and low resistance to other metabolic diseases are commonly observed during commercial broiler production. Besides this susceptibility to diseases, ascites and sudden death syndrome are now recurring issues (Kalmar et al., 2013, Wideman et al., 2013). Also, the high nutrient density feed offered ad libitum to broilers allows them to consume more calories than are required for maintenance, leading to an accumulation of abdominal fat, which is often regarded as waste (Mushtaq et al., 2014).

The response of the modern broiler chicken in growth rate, breast meat yield and feed conversion efficiency correlates strongly with both dietary energy and amino acid densities (Dozier et al., 2010; Gous et al., 2018). This emphasizes the importance of energy density when formulating diets for broiler chickens. However, improvements in feed efficiency, immune response and fat deposition require some form of dietary manipulation and planned periods of feed restriction (Eila et al., 2011; Mirshansollahi, 2013). Birds have a tendency to accumulate fat after attaining maximum growth rate, usually by 39 days old, which is followed by a decrease in growth rate after 42 days. This implies that when the growth rate drops, protein deposition also drops, whereas deposition of fat continues, and is usually high (Kessler, 2000). Therefore, if FI is not 
properly controlled, available nutrients are utilized for lipogenesis and fat accretion. Feed manipulation programmes that are aimed at changing the pattern of growth and thereby reducing storage requirements have been duly considered. These strategies can take the form of ad libitum access to less energy-dense diets or time-dependent control of daily FI. Dietary manipulations have produced positive outcomes in areas such as health and reproduction (Urdaneta-Rincon \& Leeson, 2000; Camacho et al., 2004), and control of bodyweight, fatness, and metabolic disorders (Balog et al., 2000; Rossi \& Loerch, 2003). However, there is still controversy over optimal feeding strategies for controlling carcass fat deposition, final bodyweight (BW), and feed conversion ratio (FCR).

Many studies have focused on feed restriction and dietary manipulation strategies (Santoso, 2001; Yang et al., 2013; Molapo \& Webb, 2014) and at different energy density levels (Giachetto et al., 2003). However, to the best of the authors' knowledge, no study has been conducted on changing the dietary energy density midway through the feeding period. Information on the effect of energy density in the finisher diet as a function of energy density in the starter diet is therefore unavailable. The present study aimed to investigate the effects of manipulating the energy density of the finisher diet on growth performance, haematology and carcass traits of broilers that had been fed low, optimum and high energy-density starter diets.

\section{Materials and Methods}

The ethical clearance for this study was granted by the Animal Care and Ethics Committee of The Federal University of Technology Akure, Nigeria (Ref. ONI011NAKI01).

The experiment was conducted using 378 unsexed Anak broiler chickens (Zartech Farms, Ibadan, Nigeria) that were raised from one day old to 50 days old. The feeding programme was divided into two phases. First, one-day-old chicks were randomly assigned to the three starter diets, which varied in energy density (LSE: $2786.80 \mathrm{Kcal} / \mathrm{kg}$, OSE: $3015.40 \mathrm{kcal} / \mathrm{kg}$, and HSE: $3252.20 \mathrm{Kcal} / \mathrm{kg}$ ) with seven replicates per treatment with 18 chicks per replicate. This first experiment was thus of a completely randomized design. At day 28 , each of the 27-day-old chickens was randomly reassigned to one of three finisher diets, which also varied in energy density (LFE: $2770.66 \mathrm{Kcal} . \mathrm{kg}$, OFE: $2961.74 \mathrm{Kcal} / \mathrm{kg}$, and HFE: $3150.43 \mathrm{Kcal} / \mathrm{kg}$ ) with seven replicates per treatment and six chicks per replicate. Both experimental diets were isonitrogenous.

A deep litter system of housing was used throughout the experiment, and an average of 13 hours of light was provided each day. Water and feed were offered ad libitum. The formulation of feed was done in accordance with the National Research Council (NRC, 1994) (Table 1). The feed ingredients that were used to formulate the experimental diets were analysed in triplicate for their dry matter (DM) (method 930.15), crude protein (CP) (method 988.05), ether extract (EE) (method 920.39) and crude fibre (CF) (method 978.10) (AOAC, 1990). The nitrogen content was analysed by the Kjeldahl procedure, and CP was calculated as $N \times 6.25$. Nutrient recommendations for the Anak broiler strain were used to formulate the OSE and OFE diets.

During the experimental period, the broiler chickens were weighed weekly in the morning to determine average weight gain (WG), average $\mathrm{FI}(\mathrm{FI})$ and feed to gain ratio (FCR) from days 1 to 27 and from days 28 to 50 . At the end of 50 days, three birds per replicate were selected at random, deprived of feed and water overnight, and euthanized. The birds were fully plucked, and the feet, head and wingtips removed. They were then dressed and eviscerated, and the carcass (without neck, head, lung, heart, feet, and liver) was dissected to obtain back, breast, thigh, back, wings, thighs, and drumsticks. Weighted parts, including the viscera (liver, heart, kidney, lungs, and abdominal fat) were expressed as a percentage of live weight. The thighs and drumsticks of each slaughtered bird were deboned and weighed, and the linear muscle was measured. Briefly, the thigh muscle was carefully separated into outer and inner portions and reweighed. Values were expressed as percentage of live weight. Linear measurements were also taken for the length and breadth of both portions of the thigh.

Blood samples for haematology parameters were collected from the jugular vein of each bird in heparinized bijou bottles and transported in an ice pack to the laboratory for further determination of haematological indices. The white blood cells (WBC), red blood cells (RBC), erythrocyte sedimentation rate $(E S R)$, packed cell volume (PCV), haemoglobin $(\mathrm{Hb})$ and leukogram parameters were determined on the same day of blood collection, following procedures described by Lamb (1981). The mean corpuscular haemoglobin concentration ( $\mathrm{MCHC})$, mean corpuscular haemoglobin $(\mathrm{MCH})$, and mean corpuscular volume (MCV) were calculated as described by Sastry (2004).

Data were analysed using the GLM procedure of SAS (SAS Institute Inc., 2013). The model was a one-way analysis of variance fitting treatment effects. Thus, for the starter phase there were two degrees of freedom for treatment. For the finisher phase, treatment had eight degrees of freedom. Means were compared using Duncan's multiple range test where a significant difference existed. The statement of significance was declared at $P \leq 0.05$. 
Table 1 Ingredient and nutrient composition of experimental starter and finisher diets for broiler chicks (expressed in $\mathrm{gkg}^{-1}$ of dry matter basis)

\begin{tabular}{|c|c|c|c|c|c|c|}
\hline \multirow[b]{2}{*}{ Ingredients } & \multicolumn{3}{|c|}{ Starter diet } & \multicolumn{3}{|c|}{ Finisher diet } \\
\hline & Low energy & $\begin{array}{c}\text { Optimum } \\
\text { energy }\end{array}$ & High energy & Low energy & $\begin{array}{c}\text { Optimum } \\
\text { energy }\end{array}$ & High energy \\
\hline Maize & 380.0 & 560.0 & 550.0 & 380.0 & 493.0 & 533.0 \\
\hline Maize offal & 150.0 & - & - & 200.0 & 100.0 & 85.0 \\
\hline Soybean meal & 120.0 & 140.0 & 140.0 & 120.0 & 130.0 & 140.0 \\
\hline Groundnut cake & 145.0 & 148.5 & 170.0 & 130.0 & 140.0 & 150.0 \\
\hline Brewers dried grain & 63.5 & 50.0 & & 78.0 & 60.0 & 15.0 \\
\hline Fish meal & 50.0 & 50.0 & 50.0 & - & - & - \\
\hline Oyster shell & 5.0 & 5.0 & 5.0 & 5.0 & 5.0 & 5.0 \\
\hline Rice bran & 50.0 & - & - & 50.0 & 20.0 & - \\
\hline Palm oil & - & 10.0 & 48.5 & - & 15.0 & 35.0 \\
\hline Monocalcium diphosphate & 25.0 & 25.0 & 25.0 & 25.0 & 25.0 & 25.0 \\
\hline Salt & 5.0 & 5.0 & 5.0 & 5.0 & 5.0 & 5.0 \\
\hline Lysine & 1.5 & 1.5 & 1.5 & 2.0 & 2.0 & 2.0 \\
\hline Methionine & 2.5 & 2.5 & 2.5 & 2.5 & 2.5 & 2.5 \\
\hline Premixes $^{1}$ & 2.5 & 2.5 & 2.5 & 2.5 & 2.5 & 2.5 \\
\hline \multicolumn{7}{|l|}{ Calculated composition } \\
\hline Crude protein & 224.7 & 224.5 & 224.2 & 190.8 & 190.8 & 190.7 \\
\hline Metabolizable energy, kcal/kg & 2786.8 & 3015.4 & 3252.2 & 2770.4 & 2966.3 & 3151.6 \\
\hline Crude fibre & 52.0 & 42.2 & 35.4 & 63.4 & 66.3 & 81.3 \\
\hline Calcium & 14.9 & 11.8 & 14.8 & 56.2 & 66.2 & 81.3 \\
\hline Phosphorus & 7.9 & 7.0 & 6.9 & 11.8 & 11.8 & 11.7 \\
\hline Lysine & 11.7 & 11.9 & 11.9 & 6.5 & 5.9 & 5.6 \\
\hline Methionine & 6.2 & 6.2 & 6.0 & 10.0 & 10.1 & 10.1 \\
\hline \multicolumn{7}{|l|}{ Proximate composition } \\
\hline Dry matter & 927.4 & 934.3 & 930.0 & 910.9 & 915.1 & 914.7 \\
\hline Crude protein & 214.8 & 214.8 & 215.3 & 186.1 & 186.1 & 187.2 \\
\hline Ether extract & 43.6 & 60.6 & 60.6 & 60.6 & 87.2 & 100.3 \\
\hline Crude fibre & 64.5 & 42.6 & 36.4 & 75.3 & 69.3 & 42.7 \\
\hline Ash & 81.3 & 85.1 & 89.7 & 81.0 & 83.1 & 87.1 \\
\hline Nitrogen free extract & 522.6 & 531.2 & 492.6 & 498.1 & 489.4 & 497.4 \\
\hline
\end{tabular}

\footnotetext{
${ }^{1}$ Per kg of starter diet: vitamin A: 10,000 I, vitamin D: 2,800 IU, vitamin E, 35,000 IU, vitamin K: 1,900 mg, vitamin B $12: 19$ $\mathrm{mg}$, riboflavin 7,000 mg, pyridoxine: $3,800 \mathrm{mg}$, thiamine: $2,200 \mathrm{mg}$, d-pantothenic acid, 11,000 mg, nicotinic acid: 45,000 mg, folic acid: $1,400 \mathrm{mg}$, biotin: $113 \mathrm{mg}$, copper: $8,000 \mathrm{mg}$, manganese, $64,000 \mathrm{mg}$, zinc: $40,000 \mathrm{mg}$, iron: $32,000 \mathrm{mg}$, selenium: $0.06 \mathrm{mg}$, iodine: $800 \mathrm{mg}$, cobalt: $400 \mathrm{mg}$, choline: $475,000 \mathrm{mg}$, methionine: 50,000 mg, butylated hydroxytoluene: $5,000 \mathrm{mg}$, spiramycin: $5,000 \mathrm{mg}$; and per $\mathrm{kg}$ of finisher diet: vitamin A: $10000 \mathrm{IU}$, vitamin $\mathrm{D}_{3}: 2500 \mathrm{IU}$, vitamin K: $2.4 \mathrm{mg}$, vitamin E: $44 \mathrm{IU}$, biotin: $0.1 \mathrm{mg}$, folic acid: $2.0 \mathrm{mg}$. niacin: $25 \mathrm{mg}$, calcium pantothenate: $14.32 \mathrm{mg}$, pyridoxine: $3.10 \mathrm{mg}$, riboflavin: $5 \mathrm{mg}$, thiamine: $1.2 \mathrm{mg}$, vitamin $\mathrm{B}_{12}: 10.5 \mu \mathrm{g}$, iron: $85 \mathrm{mg}$, manganese: $125 \mathrm{mg}$, copper: $7.8 \mathrm{mg}$, selenium: $0.09 \mathrm{mg}$, zinc: $60 \mathrm{mg}$, choline chloride: $5.5 \mathrm{mg}$
}

\section{Results and Discussion}

The live weight of the chicks at 27 days old increased (LSE<OSE<HSE) with dietary energy level $(P$ $<0.05)$. During the starter phase, $\mathrm{FI}$ was not affected by the varying levels of dietary energy treatment $(P>$ 0.05). Thus, FCR was affected significantly by treatment during the starter phase, with FCR being lowest in the HSE group compared with the OSE and LSE groups. The effects of dietary energy level on growth performance during the starter phase are shown in Table 2. 
When the chickens were redistributed to the finisher energy diets, the final live weight (LW) across the groups was again affected significantly (Table 3 ). Unlike the starter phase, FI was affected by energy density of the finisher diets $(P<0.05)$. Birds that were finished with LFE had higher $\mathrm{FI}$ compared with the other treatment groups. Generally, FI seemed to increase as the energy content of the feed decreased. The broiler chicks fed LSE during the starter phase and HFE at the finisher phase (LSE/HFE) had similar WG to those fed with OSE/HFE and HSE/HFE. The effect of varying dietary energy density on FCR was not significant in the finisher phase $(P>0.05)$. Total protein and energy intakes were affected in both phases $(P<0.05)$. Because the diets were isonitrogenous, protein intake increased as FI rose, whereas energy intake tended to follow the energy content of the feed.

No differences $(P>0.05)$ were observed in the ESR, PCV, RBC, $\mathrm{Hb}, \mathrm{MCHC}, \mathrm{MCH}, \mathrm{MCV}$, lymphocyte, neutrophil, monocyte, and eosinophil. Basophil was affected $(P<0.05)$ by dietary energy manipulation. However, the values were within the normal physiological range for healthy broiler chickens. The haematological indices of the broiler chickens fed the dietary finisher energy levels are shown in Table 4.

No differences were observed $(P>0.05)$ in the dressing percentage, eviscerated weight, thigh, drumstick, breast, back, head, neck, and abdominal fat. Similarly, the organ and muscle weight; heart, lungs, liver, spleen, pancreas, kidney, bursa gizzard and proventriculus, were not affected $(P>0.05)$ by the dietary finisher energy manipulation. The results of the carcass characteristics and organ weights are shown in Tables 5 and 6 , respectively.

Table 2 Growth and nutrient intake of broiler chicks fed starter diets that varied in energy density until they reached 27 days old

\begin{tabular}{lrrrrr}
\hline & \multicolumn{3}{c}{ Energy density of starter diet } & & \\
\cline { 2 - 3 } Characteristic & \multicolumn{1}{c}{ LSE } & OSE & HSE & SE & $P$-value \\
\hline Initial live weight, g/bird & 55.00 & 55.00 & 57.50 & 2.27 & 0.066 \\
Final live weight, g/bird & $800.00^{\mathrm{b}}$ & $885.00^{\mathrm{a}}$ & $920.00^{\mathrm{a}}$ & 20.22 & 0.044 \\
Total weight gained, g/bird & $745.00^{\mathrm{b}}$ & $830.00^{\mathrm{a}}$ & $862.50^{\mathrm{a}}$ & 20.58 & 0.020 \\
Total food intake, g/bird & 1617.50 & 1580.00 & 1522.50 & 57.28 & 0.081 \\
Average weight gain, g/bird/day & $26.61^{\mathrm{b}}$ & $29.64^{\mathrm{a}}$ & $30.80^{\mathrm{a}}$ & 1.53 & 0.011 \\
Average feed intake, g/bird/day & 57.77 & 56.43 & 55.45 & 2.22 & 0.075 \\
Feed conversion ratio & $2.19^{\mathrm{a}}$ & $1.91^{\mathrm{b}}$ & $1.80^{\mathrm{b}}$ & 0.13 & 0.035 \\
Nutrient intake & & & & & \\
Protein intake, g & $363.45^{\mathrm{a}}$ & $354.71^{\mathrm{a}}$ & $341.34^{\mathrm{b}}$ & 5.25 & 0.021 \\
Protein/kg bodyweight & $454.31^{\mathrm{a}}$ & $400.00^{\mathrm{b}}$ & $371.02^{\mathrm{b}}$ & 20.34 & 0.018 \\
Energy intake, Kcal & $4507.65^{\mathrm{c}}$ & $4764.33^{\mathrm{b}}$ & $4951.47^{\mathrm{a}}$ & 51.47 & 0.031 \\
Energy/kg body weight & $5634.50^{\mathrm{a}}$ & $5383.42^{\mathrm{b}}$ & $5383.03^{\mathrm{b}}$ & 58.20 & 0.026
\end{tabular}

LSE: $2786.80 \mathrm{Kcal} / \mathrm{kg}$; OSE: $3015.40 \mathrm{Kcal} / \mathrm{kg}$; and HSE: $3252.20 \mathrm{Kcal} / \mathrm{kg}$

${ }_{\mathrm{a}, \mathrm{b}, \mathrm{c}}$ Mean values followed by a similar superscript do not differ with $P=0.05$ 
Table 3 Growth performance of broiler chicken fed starter and finisher diets that varied in energy density for days 1 to 27 and 28 to 50 , respectively

\begin{tabular}{|c|c|c|c|c|c|c|c|c|c|c|c|c|}
\hline \multirow{3}{*}{ Traits } & \multirow{3}{*}{$\begin{array}{l}\text { Diets } \\
\text { Starter }^{1} \\
\text { Finisher }^{2}\end{array}$} & \multicolumn{9}{|c|}{ Dietary energy density treatments } & \multirow{3}{*}{ SEM } & \multirow{3}{*}{$P$-value } \\
\hline & & \multicolumn{3}{|c|}{ LSE } & \multicolumn{3}{|c|}{ OSE } & \multicolumn{3}{|c|}{ HSE } & & \\
\hline & & LFE & OFE & HFE & LFE & OFE & HFE & LFE & OFE & HFE & & \\
\hline \multicolumn{2}{|c|}{ Initial LW, g } & $830.0^{\mathrm{b}}$ & $825.0^{\mathrm{b}}$ & $825.0^{\mathrm{b}}$ & $912.5^{\mathrm{a}}$ & $912.5^{\mathrm{a}}$ & $910.0^{\mathrm{a}}$ & $942.5^{\mathrm{a}}$ & $942.5^{\mathrm{a}}$ & $947.5^{\mathrm{a}}$ & 35.3 & 0.04 \\
\hline \multicolumn{2}{|c|}{ Final LW, g } & $2220.0^{\mathrm{e}}$ & $2283.7^{d}$ & $2382.5^{c}$ & $2367.8^{c}$ & $2457.5^{\mathrm{ab}}$ & $2448.7^{b}$ & $2342.5^{\mathrm{cd}}$ & $2472.5^{\mathrm{ab}}$ & $2515.8^{\mathrm{a}}$ & 30.3 & 0.01 \\
\hline \multicolumn{2}{|c|}{ Total WG, $g$} & $1390.0^{d}$ & $1458.8^{b}$ & $1557.5^{\mathrm{a}}$ & $1455.2^{\mathrm{bc}}$ & $1545.0^{\mathrm{a}}$ & $1548.8^{\mathrm{a}}$ & $1400.0^{\mathrm{cd}}$ & $1530.0^{\mathrm{a}}$ & $1568.2^{\mathrm{a}}$ & 28.5 & 0.04 \\
\hline \multicolumn{2}{|c|}{ Total FI, g } & $5389.0^{\mathrm{bc}}$ & $5194.0^{d}$ & $5118.0^{d}$ & $5438.0^{\mathrm{ab}}$ & $5404.4^{\mathrm{abc}}$ & $5343.0^{c}$ & $5475.0^{\mathrm{a}}$ & $5323.0^{c}$ & $5191.0^{d}$ & 42.8 & 0.03 \\
\hline \multicolumn{2}{|c|}{$\mathrm{AFI}, \mathrm{g} / \mathrm{d}$} & 192.5 & 185.5 & 182.8 & 194.2 & 193.0 & 190.8 & 195.6 & 190.1 & 185.4 & 7.5 & 0.85 \\
\hline \multicolumn{2}{|c|}{ AWG, g/d } & 49.6 & 52.1 & 55.6 & 52.0 & 55.2 & 56.0 & 50.0 & 54.6 & 56.0 & 4.6 & 0.06 \\
\hline \multicolumn{2}{|c|}{ FCR } & 3.89 & 3.56 & 3.30 & 3.75 & 3.51 & 3.48 & 3.53 & 3.48 & 3.73 & 0.38 & 0.06 \\
\hline \multicolumn{13}{|c|}{ Nutrient intake } \\
\hline \multicolumn{2}{|c|}{ Protein Intake, g } & 1028.22 & 991.02 & 976.00 & 1037.57 & 1031.08 & 1018.91 & 1044.08 & 1015.63 & 990.44 & 29.25 & 0.058 \\
\hline \multicolumn{2}{|c|}{ Protein/kg BW } & $463.06^{a}$ & $434.09^{\mathrm{ab}}$ & $405.96^{\mathrm{bc}}$ & $438.35^{\mathrm{ab}}$ & $419.65^{b c}$ & $416.22^{\mathrm{bc}}$ & $445.817^{\mathrm{ab}}$ & $410.85^{\mathrm{bc}}$ & $393.81^{c}$ & 20.23 & 0.037 \\
\hline \multicolumn{2}{|c|}{ Energy intake, Mcal } & $149.30^{\mathrm{bc}}$ & $154.07^{\mathrm{abc}}$ & $161.30^{\mathrm{abc}}$ & $150.65^{\mathrm{bc}}$ & $160.30^{\mathrm{abc}}$ & $168.39^{\mathrm{ab}}$ & $143.81^{c}$ & $157.90^{\mathrm{abc}}$ & $172.55^{\mathrm{a}}$ & 10.32 & 0.022 \\
\hline \multicolumn{2}{|c|}{ Energy/kg BW } & 67.25 & 67.49 & 67.72 & 63.65 & 65.24 & 68.79 & 57.18 & 63.87 & 73.68 & 5.12 & 0.089 \\
\hline
\end{tabular}

TSE: $2786.80 \mathrm{Kcal} / \mathrm{kg}$; OSE: $3015.40 \mathrm{Kcal} / \mathrm{kg}$; and HSE: $3252.20 \mathrm{Kcal} / \mathrm{kg}$

LFE: $2770.66 \mathrm{Kcal} / \mathrm{kg}$; OFE: $2961.74 \mathrm{Kcal} / \mathrm{kg}$; and HFE: $3150.43 \mathrm{Kcal} / \mathrm{kg}$

BW: bodyweight, FCR: feed conversion ratio, AWG: average weight gain, AFI: average feed intake, LW: live weight

$\mathrm{a}, \mathrm{b}, \mathrm{c}, \mathrm{d}, \mathrm{e}$ W Within a row, means with a common superscript do not differ at $\mathrm{P}=0.05$ 
Table 4 Haematological indices of broiler chickens fed starter and finisher diets that varied in energy density for days 1 to 27 and 28 to 50 , respectively

\begin{tabular}{|c|c|c|c|c|c|c|c|c|c|c|c|c|}
\hline \multirow{3}{*}{ Index } & \multirow{3}{*}{$\begin{array}{l}\text { Diets } \\
\text { Starter }^{1} \\
\text { Finisher }^{2}\end{array}$} & \multicolumn{9}{|c|}{ Dietary energy density treatments } & \multirow{3}{*}{ SE } & \multirow{3}{*}{$P$-value } \\
\hline & & \multicolumn{3}{|c|}{ LSE } & \multicolumn{3}{|c|}{ OSE } & \multicolumn{3}{|c|}{ HSE } & & \\
\hline & & LFE & OFE & HSE & LFE & OFE & HSE & LFE & OFE & HSE & & \\
\hline \multicolumn{2}{|c|}{$\mathrm{ESR}, \mathrm{Mm} / \mathrm{hr}$} & 5.50 & 4.50 & 6.50 & 4.67 & 6.67 & 4.33 & 5.50 & 4.00 & 5.83 & 1.52 & 0.067 \\
\hline \multicolumn{2}{|c|}{ PCV, \% } & 23.67 & 25.67 & 24.33 & 25.33 & 24.00 & 24.00 & 25.33 & 24.00 & 24.33 & 2.58 & 0.083 \\
\hline \multicolumn{2}{|c|}{$\mathrm{RBC}, 10^{6} / \mathrm{mm}^{3}$} & 2.54 & 2.62 & 2.16 & 1.96 & 3.10 & 1.87 & 2.29 & 2.78 & 2.18 & 0.52 & 0.093 \\
\hline \multicolumn{2}{|c|}{$\mathrm{Hb}, \mathrm{g} / \mathrm{dl}$} & 7.93 & 8.50 & 8.37 & 8.43 & 8.07 & 8.03 & 8.40 & 8.00 & 8.10 & 0.49 & 0.071 \\
\hline \multicolumn{2}{|c|}{$\mathrm{MCHC}, \%$} & 33.53 & 33.13 & 33.43 & 33.30 & 33.60 & 33.47 & 33.17 & 33.33 & 33.27 & 1.78 & 0.166 \\
\hline \multicolumn{2}{|c|}{$\mathrm{MCH}, \mathrm{pg}$} & 31.23 & 32.63 & 38.63 & 45.23 & 29.13 & 43.57 & 37.47 & 29.37 & 38.30 & 9.35 & 0.159 \\
\hline \multicolumn{2}{|c|}{$M C V, U^{3}$} & 93.17 & 98.50 & 112.73 & 136.10 & 86.70 & 130.30 & 113.50 & 87.98 & 115.20 & 23.86 & 0.288 \\
\hline \multicolumn{2}{|c|}{ Lymphocyte, \% } & 59.33 & 59.33 & 60.00 & 58.67 & 62.67 & 60.33 & 57.67 & 61.67 & 61.67 & 3.25 & 0.069 \\
\hline \multicolumn{2}{|c|}{ Neutrophil, \% } & 27.67 & 28.00 & 30.33 & 27.67 & 26.67 & 27.00 & 30.67 & 28.33 & 29.33 & 1.89 & 0.079 \\
\hline \multicolumn{2}{|c|}{ Monocyte, \% } & 10.00 & 8.00 & 7.00 & 9.00 & 9.00 & 9.33 & 9.00 & 7.67 & 6.67 & 2.87 & 0.058 \\
\hline \multicolumn{2}{|c|}{ Eosinophil, \% } & 2.00 & 2.33 & 2.00 & 2.33 & 1.00 & 2.33 & 2.33 & 2.00 & 1.67 & 0.35 & 0.066 \\
\hline \multicolumn{2}{|c|}{ Basophil, \% } & $1.50^{\mathrm{b}}$ & $2.23^{\mathrm{a}}$ & $0.67^{d}$ & $2.33^{a}$ & $0.67^{d}$ & $1.00^{c}$ & $0.32^{\mathrm{e}}$ & $0.33^{e}$ & $0.67^{d}$ & 0.15 & 0.009 \\
\hline
\end{tabular}

'LSE: $2786.80 \mathrm{Kcal} / \mathrm{kg} ;$ OSE: $3015.40 \mathrm{Kcal} / \mathrm{kg}$; and HSE: $3252.20 \mathrm{Kcal} / \mathrm{kg}$

${ }^{2}$ LFE: $2770.66 \mathrm{Kcal} / \mathrm{kg}$; OFE: $2961.74 \mathrm{Kcal} / \mathrm{kg}$; and HFE: $3150.43 \mathrm{Kcal} / \mathrm{kg}$

ESR: erythrocyte sedimentation rate, PCV: packed cell volume, RBC: red blood cell, Hb: haemoglobin, MCV: mean cell volume, MCH: mean corpuscular haemoglobin, $\mathrm{MCHC}$ : mean corpuscular haemoglobin concentration

$a, b, c$ Within a row, means with a common superscript do not differ at $P=0.05$ 
Table 5 Carcass characteristics of broiler chickens fed starter and finisher diets that varied in energy density for days 1 to 27 and 28 to 50 , respectively

\begin{tabular}{|c|c|c|c|c|c|c|c|c|c|c|c|c|}
\hline \multirow{3}{*}{ Traits } & \multirow{3}{*}{$\begin{array}{l}\text { Diets } \\
\text { Starter }^{1} \\
\text { Finisher }^{2}\end{array}$} & \multicolumn{9}{|c|}{ Dietary energy density treatments } & \multirow{3}{*}{ SE } & \multirow{3}{*}{$P$-value } \\
\hline & & \multicolumn{3}{|c|}{ LSE } & \multicolumn{3}{|c|}{ OSE } & \multicolumn{3}{|c|}{ HSE } & & \\
\hline & & LFE & OFE & HFE & LFE & OFE & HFE & LFE & OFE & HFE & & \\
\hline \multicolumn{2}{|c|}{ Dressing percentage } & 91.74 & 93.23 & 91.04 & 88.35 & 91.21 & 91.79 & 94.23 & 90.80 & 91.27 & 4.86 & 0.583 \\
\hline \multicolumn{2}{|c|}{ Eviscerated weight } & 79.75 & 80.67 & 79.10 & 80.01 & 80.13 & 81.88 & 80.74 & 79.49 & 79.80 & 5.25 & 0.188 \\
\hline \multicolumn{2}{|c|}{ Thigh, g/kg LW } & 114.66 & 113.62 & 111.56 & 119.35 & 121.55 & 119.36 & 108.12 & 111.71 & 113.00 & 9.83 & 0.192 \\
\hline \multicolumn{2}{|c|}{ Drum stick, g/kg LW } & 102.0 & 104.18 & 100.18 & 109.18 & 105.86 & 98.29 & 108.47 & 98.85 & 87.52 & 8.85 & 0.081 \\
\hline \multicolumn{2}{|c|}{ Shank, g/kg LW } & 49.93 & 44.40 & 43.56 & 50.18 & 50.18 & 42.39 & 47.47 & 42.91 & 39.07 & 8.03 & 0.367 \\
\hline \multicolumn{2}{|c|}{ Wing, g/kg LW } & 87.74 & 97.90 & 87.34 & 90.71 & 90.55 & 85.18 & 92.86 & 89.64 & 84.16 & 6.05 & 0.059 \\
\hline \multicolumn{2}{|c|}{ Breast, g/kg LW } & 156.10 & 184.13 & 180.46 & 156.10 & 161.80 & 188.86 & 169.90 & 170.70 & 177.50 & 24.62 & 0.263 \\
\hline \multicolumn{2}{|c|}{ Back, g/kg LW } & 141.92 & 147.79 & 146.76 & 143.32 & 141.70 & 148.35 & 155.41 & 153.60 & 145.82 & 10.66 & 0.384 \\
\hline \multicolumn{2}{|c|}{ Head, g/kg LW } & 26.23 & 26.27 & 24.49 & 26.02 & 25.48 & 24.33 & 28.88 & 25.77 & 24.91 & 4.29 & 0.072 \\
\hline \multicolumn{2}{|c|}{ Neck, g/kg LW } & 54.52 & 46.29 & 54.51 & 55.03 & 52.56 & 58.96 & 52.03 & 55.63 & 58.80 & 6.07 & 0.086 \\
\hline \multicolumn{2}{|c|}{ Abdominal fat, g/kg LW } & 16.90 & 18.53 & 23.38 & 19.28 & 25.56 & 20.62 & 19.40 & 24.87 & 24.27 & 5.71 & 0.091 \\
\hline
\end{tabular}

LSE: $2786.80 \mathrm{Kcal} / \mathrm{kg} ;$ OSE: $3015.40 \mathrm{Kcal} / \mathrm{kg}$; and HSE: $3252.20 \mathrm{Kcal} / \mathrm{kg}$

${ }^{2}$ LFE: $2770.66 \mathrm{Kcal} / \mathrm{kg}$; OFE: $2961.74 \mathrm{Kcal} / \mathrm{kg}$; and HFE: $3150.43 \mathrm{Kcal} / \mathrm{kg}$ 
Table 6 Weights of internal organs $(\mathrm{g})$ and linear measures of specific muscles from broiler chickens fed starter and finisher diets that varied in energy density for days 1 to 27 and 28 to 50 , respectively

\begin{tabular}{|c|c|c|c|c|c|c|c|c|c|c|c|c|}
\hline \multirow{3}{*}{ Traits } & \multirow{3}{*}{$\begin{array}{l}\text { Diets } \\
\text { Starter }^{1} \\
\text { Finisher }^{2}\end{array}$} & \multicolumn{9}{|c|}{ Dietary energy density treatments } & \multirow{3}{*}{ SE } & \multirow{3}{*}{$P$-value } \\
\hline & & & LSE & & & OSE & & & HSE & & & \\
\hline & & LFE & OFE & HFE & LFE & OFE & HFE & LFE & OFE & HFE & & \\
\hline \multicolumn{2}{|l|}{ Heart } & 4.59 & 4.23 & 4.81 & 4.57 & 3.95 & 4.10 & 4.43 & 4.16 & 4.16 & 0.82 & 0.077 \\
\hline \multicolumn{2}{|l|}{ Lungs } & 5.25 & 4.47 & 4.48 & 4.47 & 4.74 & 3.96 & 4.94 & 4.40 & 4.46 & 0.95 & 0.069 \\
\hline \multicolumn{2}{|l|}{ Liver } & 16.41 & 14.53 & 14.12 & 15.17 & 13.88 & 14.43 & 15.18 & 17.17 & 12.71 & 2.42 & 0.096 \\
\hline \multicolumn{2}{|c|}{ Spleen } & 0.73 & 0.72 & 0.96 & 0.75 & 0.71 & 0.57 & 0.55 & 0.93 & 0.77 & 0.28 & 0.785 \\
\hline \multicolumn{2}{|c|}{ Pancreas } & 2.04 & 1.93 & 1.77 & 1.81 & 1.25 & 1.19 & 1.70 & 1.82 & 1.42 & 0.37 & 0.653 \\
\hline \multicolumn{2}{|c|}{ Kidneys } & 4.97 & 5.03 & 5.72 & 6.18 & 5.24 & 4.05 & 4.57 & 6.25 & 5.44 & 1.52 & 0.229 \\
\hline \multicolumn{2}{|l|}{ Bursa } & 0.84 & 0.77 & 0.90 & 0.71 & 0.86 & 0.97 & 1.19 & 1.12 & 0.79 & 0.22 & 0.328 \\
\hline \multicolumn{2}{|c|}{ Gizzard } & 24.46 & 21.12 & 25.27 & 19.59 & 20.94 & 19.12 & 24.04 & 19.67 & 20.98 & 3.75 & 0.432 \\
\hline \multicolumn{2}{|c|}{ Proventriculus } & 3.69 & 4.67 & 3.94 & 4.61 & 4.03 & 3.55 & 4.60 & 5.65 & 4.63 & 1.25 & 0.251 \\
\hline \multicolumn{2}{|c|}{ Thigh, g/Kg LW } & 41.19 & 40.91 & 40.23 & 41.40 & 47.35 & 45.44 & 39.62 & 34.95 & 42.35 & 7.05 & 0.866 \\
\hline \multicolumn{2}{|c|}{ Drumstick g/Kg LW } & 35.41 & 35.48 & 35.32 & 35.18 & 34.91 & 34.80 & 37.93 & 30.44 & 30.29 & 4.12 & 0.082 \\
\hline \multicolumn{2}{|c|}{$\mathrm{OB}, \mathrm{g} / \mathrm{Kg} \mathrm{LW}$} & 14.03 & 15.14 & 15.50 & 15.31 & 14.31 & 14.35 & 15.72 & 13.91 & 14.62 & 2.05 & 0.929 \\
\hline \multicolumn{2}{|c|}{$\mathrm{IB}, \mathrm{g} / \mathrm{Kg} \mathrm{LW}$} & 43.68 & 45.66 & 39.79 & 40.44 & 41.96 & 42.46 & 40.72 & 37.64 & 46.24 & 5.11 & 0.918 \\
\hline \multicolumn{2}{|c|}{ IB length, cm/kg LW } & 7.17 & 7.62 & 7.51 & 7.52 & 8.04 & 6.99 & 7.92 & 7.66 & 7.18 & 1.82 & 0.176 \\
\hline \multicolumn{2}{|c|}{ OB length, $\mathrm{cm} / \mathrm{kg} \mathrm{LW}$} & 8.63 & 9.09 & 8.88 & 9.31 & 9.16 & 8.90 & 9.11 & 9.48 & 8.53 & 1.23 & 0.926 \\
\hline \multicolumn{2}{|c|}{ IB breadth, cm/kg LW } & 1.52 & 1.78 & 1.88 & 1.78 & 1.77 & 1.98 & 1.86 & 1.84 & 2.06 & 0.52 & 0.267 \\
\hline \multicolumn{2}{|c|}{ OB breadth, cm/kg LW } & 3.65 & 3.52 & 3.62 & 3.50 & 3.55 & 3.62 & 3.62 & 3.37 & 3.65 & 0.68 & 0.148 \\
\hline
\end{tabular}

1LSE: $2786.80 \mathrm{Kcal} / \mathrm{kg}$; OSE: $3015.40 \mathrm{Kcal} / \mathrm{kg}$; and HSE: $3252.20 \mathrm{Kcal} / \mathrm{kg}$ 2LFE: $2770.66 \mathrm{Kcal} / \mathrm{kg}$; OFE: $2961.74 \mathrm{Kcal} / \mathrm{kg}$; and HFE: $3150.43 \mathrm{Kcal} / \mathrm{kg}$ LW: live weight; OB: outer breast, IB: inner breast 
Grains provide the main source of dietary energy for broilers. However, the energy required for birds to achieve maximum performance (NRC, 1994) necessitates the inclusion of lipids in their diets. Palm oil was added to the diets to obtain the dietary concentrations used in this study. Throughout the experimental period, no illness was observed, and the mortality rate was zero for all groups. The observed increase in weight gain with increasing dietary energy during the starter period agrees with other documented reports (Dozier et al., 2007; Ghazalah et al., 2008; Akinmoladun et al., 2016). The varying dietary energy levels affected FI in both phases. This differs from the report of Leeson et al. (1996), who fed birds diets that differed in energy level $(2700$ to $3000 \mathrm{Kcal} / \mathrm{kg}$ ) and observed no difference in Fl. Generally, modern-day broilers control FI based on the energy content of the diet. However, the low FI, especially of birds fed with dietary energy levels above the required or optimum levels in this study, could be a result of having included oil in the diet. Mateos and Sell (1982) posited that high inclusion levels of oil in broiler diets can lower FI by reducing the rate of passage through the gastrointestinal tract. However, in broilers the results on the rate of passage as affected by diets that contain oil are conflicting. The FCR during the growing phase for birds fed the low-energy starter diet was poor compared with birds fed more energy-dense diets, in agreement with Leeson et al. (1996) and Dozier et al. (2007).

Growth and FI during the finisher period were affected by the energy density of the diet that was provided. These findings disagree with results from other studies (Summers et al., 1992; Infante-Rodriguez, 2016). However, heavier weights have been reported for broilers fed low energy diets compared with a standard diet (Houshmand et al., 2011). In this study, heavier final weights were attained by birds fed more energy-dense diets. Birds fed LSE and HFE displayed more compensatory growth by showing a similar weight gain compared with the other dietary regimes. Some authors reported full weight gain recovery in the form of compensatory growth at slaughter age after feed manipulation or restriction (Demir et al., 2004; Jahanpour et al., 2015). The significant effect of dietary energy levels on FI during the finishing phase in this study disagrees with Tancharoenrat and Ravindran (2014), who reported that at higher dietary energy levels, FI was not affected. However, they reported improved feed conversion and weight gain with higher dietary energy. The report of Kim et al. (2012) on the reduction of the $\mathrm{FI}$ at higher dietary energy levels agrees with the current study. When diets were compared, weight gain was found to rise linearly with an elevation in dietary energy levels in both phases. According to Leeson et al. (1996), a rise in dietary energy consumption usually results in an improvement in weight gain. There are controversies, however, as to whether or not broiler chickens can adjust their intake of calories when fed diets with different energy levels (Leeson et al., 1996; Dozier et al., 2017; Saleh et al., 2004; Hildago et al., 2004). The redistribution of dietary finisher energy affects energy intake. This disagrees with the report of Leeson et al. (1996), who observed that energy intake was essentially the same between birds fed 3300,3100 and $2700 \mathrm{kcal} / \mathrm{kg}$ dietary metabolizable energy (ME) levels.

Consumption of dietary energy is directly related to weight gain and carcass traits. However, dietary energy manipulation did not affect the carcass traits. Values recorded for birds fed under the LSE/OFE regime group seemed to have slightly more wing, breast, back, head and neck and less abdominal fat relative to their LW than those fed under the OSE/OFE and HSE/HFE regimes. Mohebodini et al. (2009) and Onbasilar et al. (2009) found that feed manipulation did not affect the carcass traits of broiler chickens. In addition, Rosa et al. (2007) and Kim et al. (2012) reported that at slaughter age, carcass yield of broilers was not influenced under elevated dietary energy. However, an increase in yield of breast and thigh muscle, and reductions in the weight of wings, head, neck, back and legs and drumstick for birds fed high energy and protein diets have also been reported (Marcu et al., 2012, 2013). Contrarily, supplementation of extra energy and protein to broiler's diet reportedly improve carcass yield in female broilers. About $8.6 \%, 9.5 \%$ and $9 \%$ increase in the starter $(3026 \mathrm{Kcal} / \mathrm{kg})$, grower $(3142 \mathrm{Kcal} / \mathrm{kg})$ and finisher $(3196 \mathrm{Kcal} / \mathrm{kg})$ dietary ME, respectively, improved breast and drumstick for both genders of broiler chickens (Marcu, 2013). However, Lee and Leeson (2001) noted that when there was a compensatory growth in birds following a period of under-nutrition, the additive effect on the carcass characteristics of economic importance was not significant. Fat deposition in broilers has been reported to positively correlate with elevated dietary energy levels (Leeson et al., 1996). However, the values obtained in this study were not affected by dietary finisher energy manipulation. This result agrees with Giachetto et al. (2003), who failed to show a significant effect of the interaction between feed manipulation and energy levels on carcass fat. Most studies have reported the absence of abdominal fat response to dietary manipulation (Saleh et al., 2005; Mohebodini et al., 2009). Conversely, others have reported a reduction (Boostani et al., 2009) or even an increase (Zhan et al., 2007) in abdominal fat percentage. When supplemented with lysine, Zhao et al. (2008) noted that higher dietary energy increases breast, carcass, abdominal fat, and thigh. Feeding broiler chickens with extra energy and amino-acid levels has been reported to increase abdominal fat deposition, while the weight of carcass cuts remained unaffected (Wang et al., 2014). However, carcass fat deposition was found to be confounded with protein and amino acids intake (Jackson et al., 1982; Leeson et al., 1996). When Fl increases, naturally the 
crude protein intake will increase, and this may reduce carcass fat deposition. Summer et al. (1992) affirmed that deposition of excess dietary energy is confined to the abdomen and can slow processing procedures because carcass evisceration is slower for birds with excessive abdominal fat.

Values obtained for the internal organs concur with the report of Rabie et al. (2017), who observed insignificant effects of various energy levels $(2900,3000$, and $3100 \mathrm{kcal} / \mathrm{kg} \mathrm{ME})$ on weights of gizzard, liver, and heart in 14-week-old cockerels. Carcass traits of broiler chicks were not influenced by elevated dietary energy levels (Rabie et al., 2010). Carcass cuts and yield were not affected by dietary ME levels in starter $(12.55-13.39 \mathrm{MJ} / \mathrm{kg}$ ) and finisher (12.79 - $13.8 \mathrm{MJ} / \mathrm{kg}$ ) phases in a study by Corduk et al. (2007). In the report of Araujo (1982), the effect of energy levels (3200 vs $3600 \mathrm{kcal} \mathrm{ME} / \mathrm{kg}$ ) did not influence the liver, heart, and gizzards.

Muscle and connective tissues are the most abundant tissues in meat, and their properties and relative proportions are responsible for the leanness and quality of meat (Listrat et al., 2016). Muscle fibre, and biochemical and structural characteristics can be manipulated independently by intrinsic and extrinsic factors to improve meat quality (Joo et al., 2013; Listrat et al., 2016). However, the findings in this study did not reveal any particular trend in terms of linear muscle dimensions or morphometrics with dietary finisher energy manipulation.

Analysis of blood samples can be used to probe for particular metabolites in an animal's body (Doyle, 2006). The haematological values that were obtained in this study were not affected by the dietary energy levels. This implies that protein digestion had not been interfered with. These results agree with those of Jahanpour et al. (2013), who investigated the effect of quantitative feed restriction on the blood metabolites of broiler chickens. However, the blood indices obtained in this study differ from the report of Dairo et al. (2010), who observed a significant effect on the haematology of birds fed a high-energy-low-protein diet. Similarly, the PCV and $\mathrm{Hb}$ of slow-growing chicks fed various levels of dietary energy have been shown to be affected during exposure to heat stress (Attia et al., 2011). The leukogram parameters (lymphocytes, neutrophils, eosinophils) reported in this study were not affected either, implying that phagocytosis, the ability to fight disease invasions, was not impaired by the diets. All haematological indices that were recorded in this study were within the recommended range for normal and healthy chickens (Mitruka \& Rawnsley, 1977; Olorode et al., 1996).

\section{Conclusions}

Broiler chickens would still be able to attain market weight when fed with a less dense nutrient diet in the starter phase, followed by a high energy diet in the finisher phase. Further, dietary finisher energy manipulation did not compromise the health status of the chicken. However, given the mixed reports regarding body composition, an increase in fatness is possible after feeding a less energy dense diet in the starter phase.

\section{Acknowledgements}

Authors are grateful to Govan Mbeki Research and Development Centre (GMRDC), University of Fort Hare, South Africa, for providing the financial assistance to publish this manuscript.

\section{Authors' Contributions}

OFA: conceptualization, methodology, writing and original draft preparation; ABF: analysis, writing, review and editing; OFA and ABF: funding acquisition

\section{Conflicts of Interest Declaration}

The authors declare no conflict of interest.

\section{References}

Akinmoladun, O.F., Onibi, G.E., Babalola, K.O., Lomuwagun, A.T. \& Fabode, A.F., 2016. Growth performance, haematology and carcass characteristics of broiler chicks fed diets with varying energy levels. American Journal of Experimental Agriculture 10(5), 1-6.

AOAC, 1990. Official methods of analysis. 16th edition. Association of Official Analytical Chemists, Arlington, VA.

Araújo, L.F., 1998. Avaliação do desempenho e rendimento de carcaça de frangos de cortesubmetidos a dietas com altos níveis de energia, metionina+cistina e lisinanafase final de criação. Dissertação, Jaboticabal (SP), UniversidadeEstadualPaulista.

Attia, Y.A., Hassan, R.A., Tag El-Din, A.E. \& Abou-Shehema, B.M., 2011. Effect of ascorbic acid or increasing metabolizable energy level with or without supplementation of some essential amino acids on productive and physiological traits of slow-growing chicks exposed to chronic heat stress. J Anim. Phys. Anim. Nutr. 95, 744-755.

Balog, J.M., Anthony, N.B., Cooper, M.A., Kidd, B.D., Huff, G.R., Huff, W.E. \& Rath, N.C., 2000. Ascites syndrome and related pathologies in feed restricted broilers raised in a hypobaric chamber. Poult. Sci. 79, 318-323. 
Boostani, A., Ashayerizadeh, A., MahmoodianFard, H.R. \& Kamalzadeh, A., 2010. Comparison of the effects of several feed restriction periods to control ascites on performance, carcass characteristics and hematological indices of broiler chickens. Braz. J. Poultry Sci. 12,171-177.

Camacho, M.A., Sua'rez, M.E., Herrera, J.G., Cuca, J.M. \& Garcı'a-Bojalil, C.M., 2004. Effect of age of feed restriction and microelement supplementation to control ascites on production and carcass characteristics of broilers. Poult. Sci. 83, 526-532.

Corduk, M., Ceylan, N. \& Ildiz, F., 2007. Effects of dietary energy density and I-carnitine supplementation on growth performance, carcass traits and blood parameters of broiler chickens. S. Afr J. Anim. Sci. 37, 65-73.

Dairo, F.A.S., Adesehinwa, A.O.K., Oluwasola, T.A. \& Oluyemi, J.A., 2010. High and low dietary energy and protein levels for broiler chickens. Afr. J. Agric. Res. 5(15), 2030-2038.

Demir, E., Sarica, S., Sekeroglu, A., Ozcan, M.A. \& Seker, Y., 2004. Effects of early and late feed restriction or feed withdrawal on growth performance, ascites and blood constituents of broiler chickens. Acta Agr. Scand. A 54, 152-158.

Doyle, D. William Hewson [1739-74], 2006. The father of haematology. British Journal of Haematology 133(4), 375-381.

Dozier, W.A. III., Kidd, M.T., Corzo, A. \& Branton, S.L., 2007. Dietary apparent metabolizable energy and amino acid density effects on growth an. carcass traits of heavy broilers. J. Appl. Poult. Res., 16, 192-205.

Dozier. W.A., Corzo, A., Kidd, M.T., Tillman, P.B., McMurtry, J.P. \& Branton, S.L., 2010. Digestible lysine requirements of male broilers from 28 to 42 days of age. Poult Sci. 89(10), 2173-82.

Eila, N., Lavvaf, A.G. \& Farahvash, T., 2011. Comparison of different feed restriction methods on growth performance of broilers. Res. Opin. Anim. Vet. Sci. 1, 686-688.

Ghaffari, M., Shivazad, M., Zaghari, M. \& Taherkhani, R., 2007. Effects of different levels of metabolizable energy and formulation of diet based on digestible and total amino acid requirements on performance of male broiler. Int. J. Poult. Sci. 6, 276-279.

Ghazalah, A.A., Abd-Elsamee, M.O. \& Ali, A.M., 2008. Influence of dietary energy and poultry fat on the response of broiler chicks to heat stress. Int. J. Poult. Sci. 7(4), 355- 359.

Giachetto, P.F., Guerreiro, E.N., Ferro, J.A., Ferro, M.I.T., Furlan, R.L. \& Macari, M., 2003. Performance and hormonal profile in broiler chickens fed with different energy levels during post restriction period. Pesqui. Agropecu. Bras., 38, 697-702.

Gous, R.M., Faulkner, A.S. \& Swatson, H.K., 2018. The effect of dietary energy: protein ratio, protein quality and food allocation on the efficiency of utilisation of protein by broiler chickens. Brit. Poultry Sci. 59(1), 100-109.

Havenstein, G.B., Ferket, P.R. \& Qureshi, M.A., 2003. Growth, livability, and feed conversion of 1957 versus 2001 broilers when fed representative 1957 and 2001 broiler diets. Poult. Sci. 1500-1508.

Hildago, M.A., Dozier, W.A.III., Davis, A.J. \& Gordon, R.W., 2004. Live performance and meat yield responses to progressive concentrations of dietary energy at a constant metabolizable energy-crude protein ratio. J. Appl. Poult. Res. 13, 319-327.

Houshmand, M., Azhar, K., Zulkifli, I., Bejo, M.H. \& Kamyab, A., 2011. Effects of nonantibiotic feed additives on performance, nutrient retention, gut $\mathrm{pH}$, and intestinal morphology of broilers fed different levels of energy. J. Appl. Poult. Res. 20, 121-128.

Infante-Rodriguez, F., Salina-Chavira, J., Montano-Gomez, M.F., Manriquez-Nunez, O.M., Gonzalez-Vizcarra, V.M. Guevara, O.F. \& Ramirez De Leon, J.A., 2016. Effect of diets with different energy concentrations on growth performance, carcass characteristics and meat chemical composition of broiler chickens in dry tropics. Springer Plus 5, 1937.

Jackson, S., Summers, J.D. \& Lesson, S., 1982. Effect of dietary protein and energy on broiler carcass composition and efficiency of nutrient utilization. Poult. Sci. 61, 2224-2231.

Jahanpour, H., Seidavi, A., Qotbi, A.A.A. \& Payan-Carreira, R., 2013. Effects of two levels of quantitative feed restriction for a 7-or 14- days period on broilers blood parameters. Acta Sci. Vet. 41, 1-11.

Jahanpour, H., Seidavi, A., Qotbi, A.A.A., Van Den Hoven, R., Silva, S.R., Laudadio, V. \& Tufarelli, V., 2015. Effects of the level and duration of feeding restriction on carcass components of broilers. Arch. Anim. Breed. 58, 99-105.

Joo, S.T., Kim, G.D., Hwang, Y.H. \& Ryu, Y.C., 2013. Control of fresh meat quality through manipulation of muscle fiber characteristics. Meat Sci. 95, 828-836.

Kalmar, I.D., Vanrompay, D. \& Janssens, G.P.J., 2013. Broiler ascites syndrome: Collateral damage from efficient feed to meat conversion. Vet. J. 197, 169-174.

Kessler, A.M., SnizekJúnior, P.N. \& Brugalli, I., 2000.Manipulação da quantidade de gorduranacarcaça de frangos. In: ConferênciaApinco de Ciência e TecnologiaAvícolas; Campinas, São Paulo. Brasil. p. 107-133.

Kim, J.S., Kwon, J.T., Harim, L., Kim, J.H., Oh, S.K., Lee, B.K., Zheng, L., Konkuk Jung, M.S., An, B.K. \& Kan, C.W., 2012. Performance and carcass characteristics of two different broiler strains by different levels of metabolizable energy. Korean J. Poult. Sci. 39, 195-205.

Lamb, G.N. (ed), 1981. Manual of veterinary technician. CIBA-Geigy, Kenya. Pp. 92-109.

Lee, K.H. \& Leeson, S., 2001. Performance of broilers fed limited quantities of feed or nutrients during seven to fourteen days of age. Poultry Sci. 80, 446-454.

Leeson, S., Caston, L. \& Summers, J.D., 1996. Broiler response to diet energy. Poult. Sci. J. 75, 529-535.

Listrat, A., Lebret, B., Louveau, I., Astruc, T., Bonnet, M., Lefaucheur, L., Picard, B. \& Bugeon, J., 2016. How muscle structure and composition influence meat and flesh quality. Scientific World J. 2016, 1-14.

Longo, F.A., 2000. Estudo do metabolismoenergético e do crescimentoemfrangos de corte. Dissertação, Jaboticabal (SP), UniversidadeEstadualPaulista. 
Marcu, A., Vacaru-Opriş, I., Marcu, A., Nicula, M., Dronca, D. \& Kelciov, B., 2012. Effect of different levels of dietary protein and energy on the growth and slaughter performance at Hybro PN+ broiler chickens. Pap. Anim. Sci. Biotechnol. 45, 424-431.

Marcu, A., Vacaru-Opriş, I., Dumitrescu, G., Marcu, A., Petculescu, C.L., Nicula, M., Dronca, D. \& Kelciov, B., 2013. Effect of diets with different energy and protein levels on breast muscle characteristics of broiler chickens. Pap. Anim. Sci. Biotechnol. 46, 333-340

Mateos, G.G. \& Sell, J.L., 1982. Rate of food passage (time transit) as influenced by level of supplemental fat. Poultry Science 61, 94-100.

Mirshamsollahi, A., 2013. Effect of different food restriction on performance and carcass characteristics of Arian and Ross broiler chicks. Int. J. Agric. Res. Rev., 3, 495-501.

Mitruka, B.M. \& Rawnsley, M.,1977. Clinical, biochemical and haematological reference values in normal experimental animals. J. Clinical Pathology 42(1), 65-70.

Mohebodini, H., Dastar, B., Shams Sharg, M. \& Zerehdaran, S., 2009. The comparison of early feed restriction and meal feeding on performance, carcass characteristics and blood constituents of broiler chickens. J. Anim. Vet. Adv. 8:2069-2074

Molapo, S.M. \& Webb, E.C., 2014. Effect of restricted feeding on the carcass characteristics of Koekoek chickens. Res. Opin. Anim. Vet. Sci. 299-304.

Mushtaq, M.M.H., Pasha, T.N., Mushtaq, T., Akram, M., Mahmood, S., Farooq, U. \& Parvin, R., 2014. Growth, water intake, litter moisture, carcass and physiological traits of broiler chickens fed varying levels and sources of potassium under phase feeding system. Livest. Sci. 159, 61-66.

Nangsuay, A., Molenaar, R., Meijerhof, R., van den Anker, I., Heetkamp, M.J.W., Kemp, B. \& Van den Brand, H., 2015. Differences in egg nutrient availability, development, and nutrient metabolism of broiler and layer embryos. Poult. Sci. 94: 415-442

NRC, 1994. Nutrient requirements of poultry. 9th revised edition. National Academy Press, Washington, DC, USA.

Olorode, B.R.,Onifade, A.A., Okpara, A.O. \& Babatunde, G.M., 1996. Growth, nutrient retention, hematology and serum chemistry of broiler chickens fed shea butter cake or palm kernel cake in the humid tropics. J. Appl. Anim. Res. 10, 173-180.

Onbasılar, E.E., Yalçın, S., Torlak, E. \& Özdemir, P., 2009. Effects of early feed restriction on live performance, carcass characteristics, meat and liver composition, some blood parameters, heterophil-lymphocyte ratio, antibody production and tonic immobility duration. Trop. Anim. Health Pro. 41, 1513-1519.

Rabie, M.H., El Sherif, K.H., Abd El-Khalek, A.M. \& El-Gamal, A.A.A., 2017. Effect of dietary energy and protein on growth performance and carcass traits of Mamourah cockerels. Asian Journal of Animal and Veterinary Advances $12,142-151$.

Rabie, M.H., Ismail, F.S.A. \& Sherif, S.K., 2010. Effect of dietary energy level with probiotic and enzyme addition on performance, nutrient digestibility and carcass traits of broilers. Egypt. Poult. Sci. J. 30, 179-201

Rosa, P.S., FariaFilho, D.E., Dahlke, F., Vieira, B.S., Macari M. \& Furlan, R.L. 2007.Effect of energy intake on performance and carcass composition of broiler chickens from two different genetic groups. Braz. J. Poult. Sci. 9, 117-122.

Rossi, J.E. \& Loerch. S.C., 2003. Effects of duration of feed restriction on carcass composition of Leghorn cockerels. J. Appl. Anim. Res. 2, 49-57.

Saleh, E.A., Watkins, S.E. \& Waldroup, A.L., 2004. Consideration for dietary nutrient and energy feeding programs for growing large male broilers chickens for further processing. Int. J. Poult. Sci. 3, 11-16.

Saleh, E.A., Watkins, S.E., Waldroup, A.L. \& Waldroup, P.W., 2005. Effects of early quantitative feed restriction on live performance and carcass composition of male broilers grown for further processing. J. Appl. Poultry Res. 14:8793.

Santoso, I., 2001. Effect of early feed restriction on growth, fat accumulation and meat composition in unsexed broiler chickens. Asian-Aust. J. Anim. Sci. 4(11),1585-1561.

SAS Institute Inc., 2013. SAS System for Windows. Release 9.4.

Sastry, G.A., 2004. Veterinary clinical pathology. CBS, 11 Darya Ganj, New Delhi, India. Pp. 1-41.

Summers, J.D., Spratt, D. \& Atkinson, J.L., 1992. Broiler weight gain and carcass composition when fed diets varying in amino acid balance, dietary energy and protein level. Poult. Sci. 71, 263-273.

Tancharoenrat, P. \& Ravindran, V., 2014. Influence of tallow and calcium concentrations on the performance and energy and nutrient utilization in broiler starters. Poult. Sci. 93, 1453-1462

Tůmová, E., Skřivan, M., Skřivanová, V.\& Ka-Cerovská, L., 2002. Effect of early feed restriction on growth in broiler chickens, turkeys and rabbits. Czech J. Anim. Sci. 47, 418-428.

Tumova, E. \& Teimouri, A., 2010. Fat deposition in the broiler chicken: A review. Sci. Agr. Bohem. 41, 121-128.

Urdaneta-Rincon, M. \& Leeson, S., 2000. Quantitative and qualitative feed restriction on growth characteristics of male broiler chickens. Poult. Sci. 81, 679-688

Wang, X., Peebles, E.D. \& Zhai, W., 2014. Effects of protein source and nutrient density in the diets of male broilers from 8 to 21 days of age on their subsequent growth, blood constituents, and carcass compositions. Poult. Sci. 93,4631474.

Wideman, R.F., Rhoads, D.D. \& Erf, G.F., Anthony, N.B., 2013. Pulmonary arterial hypertension (ascites syndrome) in broilers: A review. Poult. Sci. 92, 64-83.

Yang, H., Yang, Z., Wang, Z., Wang, W., Huang, K., Fan, W. \& Jia, T., 2015. Effects of early dietary energy and protein dilution on growth performance, nutrient utilization and internal organs of broilers. Italian Journal of Animal Science 14(2), 163-171. 
Zhan, X.A., Wang, M., Ren, H., Zhao, R.Q., Li, J.X. \& Tan, Z.L., 2007. Effect of early feed restriction on metabolic programming and compensatory growth in broiler chickens. Poult. Sci. 86, 654-660.

Zhao, L.H., Ma, Q.G., Chen, X.D., Hu, X.X. \& Ji, C., 2008. Effects of dietary energy levels and lysine levels on performance and carcass characteristics in Arbor Acres Broilers. Chin. J. Anim. Sci. 44, 35-40. 